\title{
Plasmonic Oligomers with Tunable Conductive Nanojunction
}

Xiaoyan Li, ${ }^{1}$ Jieli Lyu, ${ }^{1}$ Claire Goldmann, ${ }^{1}$ Mathieu Kociak, ${ }^{1}$ Doru Constantin, ${ }^{1 *}$ Cyrille Hamon $^{1 *}$

${ }^{1}$ Laboratoire de Physique des Solides, CNRS, Univ. Paris-Sud, Université Paris-Saclay, 91405 Orsay Cedex, France.

\section{Contents}

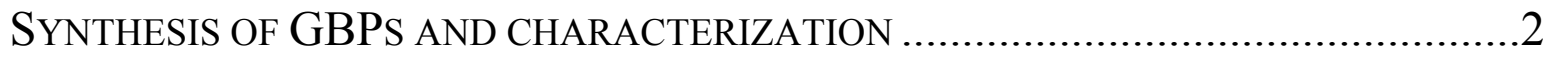

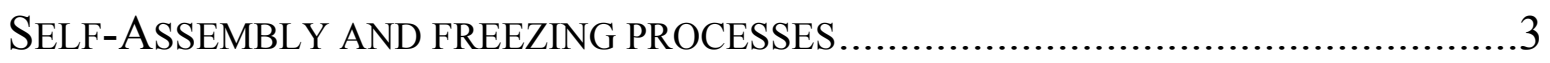

METAL DEPOSITION AT THE GBPS OLIGOMERS JUNCTION .................................4

CHARACTERIZATION TECHNIQUES ................................................................

BOUNDARY ELEMENT METHOD (BEM) SIMULATION DETAILS ............................5

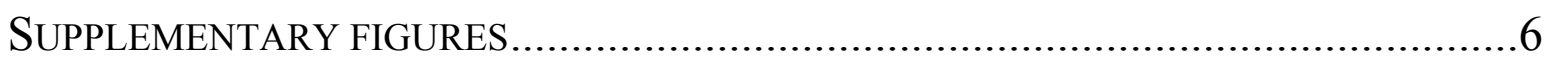

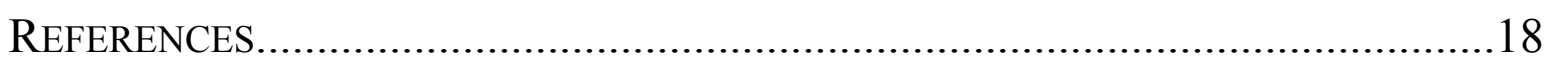




\section{Synthesis of GBPs and characterization}

Materials: All the starting materials were obtained from commercial suppliers and used without further purification: hexadecyltrimethylammonium bromide (CTAB, $\geq 99 \%$ ), hexadecyltrimethylammonium chloride (CTAC, 25 wt \% in $\mathrm{H}_{2} \mathrm{O}, 90 \%$ ), hydrogen tetrachloroaurate trihydrate $\left(\mathrm{HAuCl}_{4} \cdot 3 \mathrm{H}_{2} \mathrm{O}, \geq 99.9 \%\right)$, potassium hexachloropalladate $\left(\mathrm{K}_{2} \mathrm{PdCl}_{6}, \geq 99 \%\right)$, silver nitrate $\left(\mathrm{AgNO}_{3}, \geq 99.0 \%\right)$, Chloroplatinic acid hexahydrate $\left(\mathrm{H}_{2} \mathrm{PtCl}_{6}\right.$, $\left.6 \mathrm{H}_{2} 0\right)$ L-ascorbic acid (AA, $\geq 99 \%$ ), sodium borohydride $\left(\mathrm{NaBH}_{4}, 99 \%\right)$, trisodium citrate ( $\geq 99.0 \%)$. Magnesium sulfate $\left(\mathrm{MgSO}_{4}, \geq 95 \%\right)$ was purchased from Prolabo. Water purified by reverse osmosis with a resistivity (>15 $\mathrm{M} \Omega . \mathrm{cm})$ was used in all experiments.

Synthesis of Gold Pentatwinned Seeds: Synthesis of gold pentatwinned seeds was adapted from the literature. ${ }^{1,2} \mathrm{In}$ a $50 \mathrm{~mL}$ Erlenmeyer flask, $16.5 \mathrm{~mL}$ of a $60.6 \mathrm{mM}$ CTAC solution was constituted in which $200 \mu \mathrm{L}$ of $25 \mathrm{mM} \mathrm{HAuCl}_{4}$ and $2 \mathrm{~mL}$ of $50 \mathrm{mM}$ sodium citrate was added. The solution was kept in a water bath with at $30^{\circ} \mathrm{C}$ for 10 minutes under gentle stirring. Then, $1.2 \mathrm{~mL}$ of a $10 \mathrm{mM} \mathrm{NaBH}_{4}$ solution was injected under vigourous stirring. A reddish solution was obtained after aging the samples during 5 days at $40^{\circ} \mathrm{C}$. The seed solution can be stored for at least one month at room temperature.

Synthesis of Gold Bipyramids: In a $250 \mathrm{~mL}$ Erlenmeyer flask, growth solution was prepared by the successive addition of $100 \mathrm{~mL}$ of $0.1 \mathrm{M} \mathrm{CTAB}, 1 \mathrm{~mL}$ of $10 \mathrm{mM} \mathrm{AgNO}_{3}, 2 \mathrm{~mL}$ of 25 $\mathrm{mM} \mathrm{HAuCl}_{4}$ and $2 \mathrm{~mL}$ of $1 \mathrm{M} \mathrm{HCl}$. Then, $800 \mu \mathrm{L}$ of a $100 \mathrm{mM} \mathrm{AA}$ solution was added under vigourous stirring, followed by the addition of $900 \mu \mathrm{L}$ of seed solution. The mixture was left undisturbed at $30^{\circ} \mathrm{C}$ for at least $4 \mathrm{~h}$. After synthesis, particles were purified by at least two cycles of centrifugation and pellet redispersion in $[\mathrm{CTAB}]=1.8 \mathrm{mM}$ in order to obtain a dispersion of $16 \mathrm{mM}$ GBPs (in $\mathrm{Au}^{0}$ ) and $1.8 \mathrm{mM} \mathrm{CTAB}$. The GBPs dispersion can be stored for months in ambient conditions. The transverse surface plasmon resonance band (TSPR) and longitudinal surface plasmon resonance band (LSPR) of the GBP suspension are located around 
$510 \mathrm{~nm}$ and $696 \mathrm{~nm}$ respectively (Figure S1-A). The high shape yield of GBPs versus other morphologies can be clearly seen in the TEM images and is confirmed the high intensity of the LSPR compared to the TSPR band ( $\mathrm{I}_{\mathrm{LSPR}} / \mathrm{I}_{\mathrm{TSPR}}$ of 4.2$)$.

\section{Self-Assembly and freezing processes}

Polymerization was induced by diluting $31 \mu \mathrm{L}$ of the GBPs dispersion with a $2 \mathrm{~mL}$ of $1 \mathrm{mM}$ $\mathrm{MgSO}_{4}$ solution in a cuvette, yielding a GBPs dispersion comprising $\left[\mathrm{Au}^{0}\right]=0.25 \mathrm{mM}$, $[\mathrm{CTAB}]=28 \mu \mathrm{M}$ and $\left[\mathrm{MgSO}_{4}\right]=1 \mathrm{mM}$. Self-assembly was monitored by time resolved UV/Vis spectroscopy (See Figure 1B in the manuscript). The polymerization rate can be tuned over a wide range by varying the $\mathrm{CTAB}$ concentration during the self-assembly process while keeping fixed other parameters such as the sulfate and GBPs concentration (Figure S2). No evolution of the optical properties was noted for CTAB concentration above $100 \mu \mathrm{M}$. Conversely, GBPs precipitated within $1 \mathrm{~h}$ at a $\mathrm{CTAB}$ concentration of $4.5 \mu \mathrm{M}$. Those results suggest a modification of the chemical environment of the GBPs in this CTAB concentration range that can "activate" the GBPs for their self-assembly mediated with sulfate ion. Note that we did not observe any modification of the optical properties (over 3 days) when the GBPs were diluted with water under otherwise identical conditions (See Figure S3). Moreover, the self-assembly process can be freeze at any stage of the reaction by adding CTAB or CTAC in solution, resulting in stable oligomers dispersion (Figure S4). It is worth noting that similar kinetic arrest was observed when adding CTAB at varying concentrations in the 1-100 $\mathrm{mM}$ range.

\section{Metal deposition at the GBPs oligomers junction}

Metallic junction were engineered with silver or palladium by seed mediated growth. In a typical experiment, $1 \mathrm{~mL}$ of stabilized oligomers $\left(0.25 \mathrm{mM} \mathrm{Au}{ }^{0}, 10 \mathrm{mM} \mathrm{CTAC}\right)$ was supplemented with $5 \mu \mathrm{L}$ of $10 \mathrm{mM}$ metallic precursor $\left(\mathrm{AgNO}_{3}, \mathrm{~K}_{2} \mathrm{PdCl}_{6}, \mathrm{H}_{2} \mathrm{PtCl}_{6}\right)$ and $5 \mu \mathrm{L}$ of $40 \mathrm{mM}$ AA solution. This corresponds to a molar ratio between the metallic precursor and gold of 0.2. The molar ratio between the AA and metallic precursor was fixed to 4 in all experiments but the molar ratio between the metallic precursor and GBPs can be varied to tune the thickness 
of the junction (Figure S5). After mixing, the reaction vials were kept at $60^{\circ} \mathrm{C}$ during $1 \mathrm{~h}$. After the synthesis, connected oligomers were purified from the excess of reactant by at least one centrifugation steps $(1500 \mathrm{~g}, 30 \mathrm{~min})$ and pellet redispersion in a solution of $1 \mathrm{mM} \mathrm{CTAC}$ at a final gold concentration of $0.25 \mathrm{mM}$ in all samples.

\section{Characterization techniques}

UV/Vis spectroscopy: UV/Vis/NIR absorption spectra were registered using a Cary $5000 \mathrm{UV}$ Vis-NIR. All experiments were carried out at room temperature, using disposable polystyrene cuvettes with optical paths of $1 \mathrm{~cm}$.

Transmission electron microscopy: TEM images were obtained with a JEOL 1400 transmission electron microscope, operating at an acceleration voltage of $120 \mathrm{kV}$. NPs deposition was performed on carbon coated grid. The plasmonic response of the nanostructures were studied using electron energy loss spectroscopy in the NION CHROMATEM monochromated Cs-corrected scanning transmission electron microscope. The $15 \mathrm{~nm}$ thick $\mathrm{Si}_{3} \mathrm{~N}_{4}$ support membrane grids were used for NPs deposition in order to provide a carbon-free background at the low energy range for a better observation of the plasmonic peaks. The EELS spectrum images were acquired at $60 \mathrm{keV}$, with a spatial resolution of a few nanometers and a typically energy resolution of 20-30 meV. The EELS spectra (such as Figure $3 \mathrm{~b}, 4 \mathrm{~b}, \mathrm{~S} 11 \mathrm{~b}$, $\mathrm{S} 12 \mathrm{~b}, \mathrm{~S} 13 \mathrm{~b})$ were extracted by summing a few hundreds of spectra in a region of interest close to the nanostructures and normalize by the intensity of the zero-loss peak (ZLP).

\section{Boundary Element Method (BEM) simulation details}

Simulated extinction spectra were calculated by the boundary element method (BEM), using the MNPBEM toolbox developed in the MATLAB environment. ${ }^{3}$ The GBPs are described as a gold bicone with $35.5 \mathrm{~nm}$ in length and $12.5 \mathrm{~nm}$ in base-width, terminated with two spherical caps. The truncation of the bicone was adjusted in the simulations to fit the experimental spectra and we observed that the L-LSPR red shift as the sharpness of the bipyramid increase. For 
simulations, each particles were modeled with a triangular mesh consisting of 1330 faces and were spaced by $1 \mathrm{~nm}$ from each other in a tip-to-tip arrangement (Figure S6A). Simulations were performed to solve the full Maxwell's equations (including retardation effects). The dielectric properties of gold were taken from E. Palik and G. Ghosh. ${ }^{4}$ EELS spectra and EELS MAPs were obtained using an arbitrary value for the dielectric constant (i.e. 1.2) in order to take into account the $\mathrm{Si}_{3} \mathrm{~N}_{4}$ membrane/NPs interaction. Even with this correction, the peaks are systematically blue shifted in respect to the experiment, indicating that the membrane/NPs interaction is imperfectly taken in consideration in the simulation. The energy of the simulated electron beam was set to the experimental accelerating voltage of the TEM $(60 \mathrm{kV})$. Connected oligomers with a silver nanojunction were approximated as single continuous gold NPs (Figure S9B) as the small distances between interfaces when considering silver junction were otherwise introducing numerical artifacts. Although a reasonable agreement between experiment and simulation was observed in the case of a silver junction, this approximation do not permit to get simulation for connected oligomers with a palladium junction. 
Supplementary figures
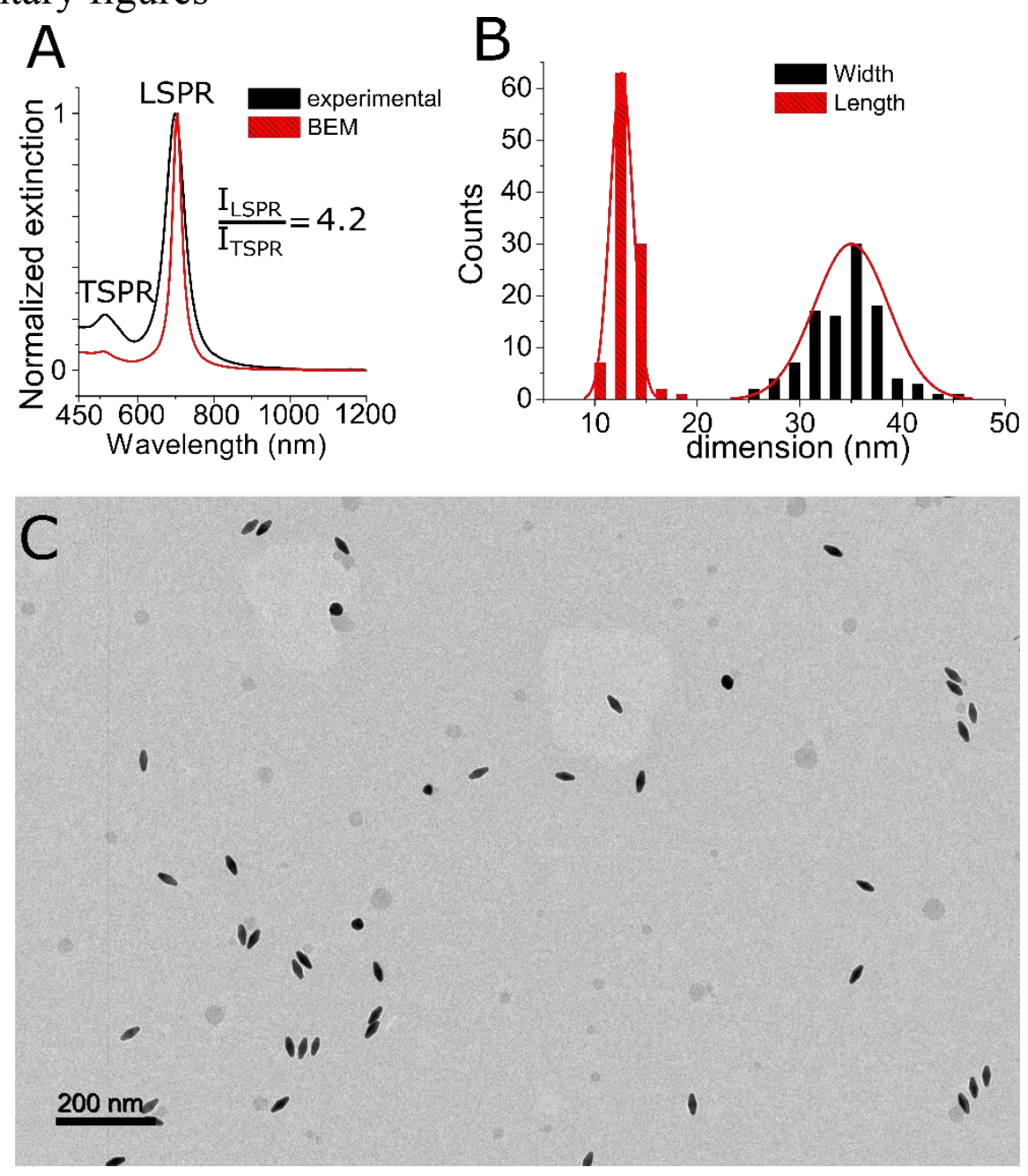

Figure S1: Characterization of the GBPs. A) UV-Vis spectra of the GBPs dispersion (black) and corresponding simulated spectra (red). For BEM simulation, the dimension of the particles was determined from TEM image analysis. B) Size histogram of the GBPs $35.0 \pm 4.3 \mathrm{~nm}$ long; $12.5 \pm 1.4 \mathrm{~nm}$ base-width determined from TEM image analysis. C) Representative TEM image of dispersed GBPs. 

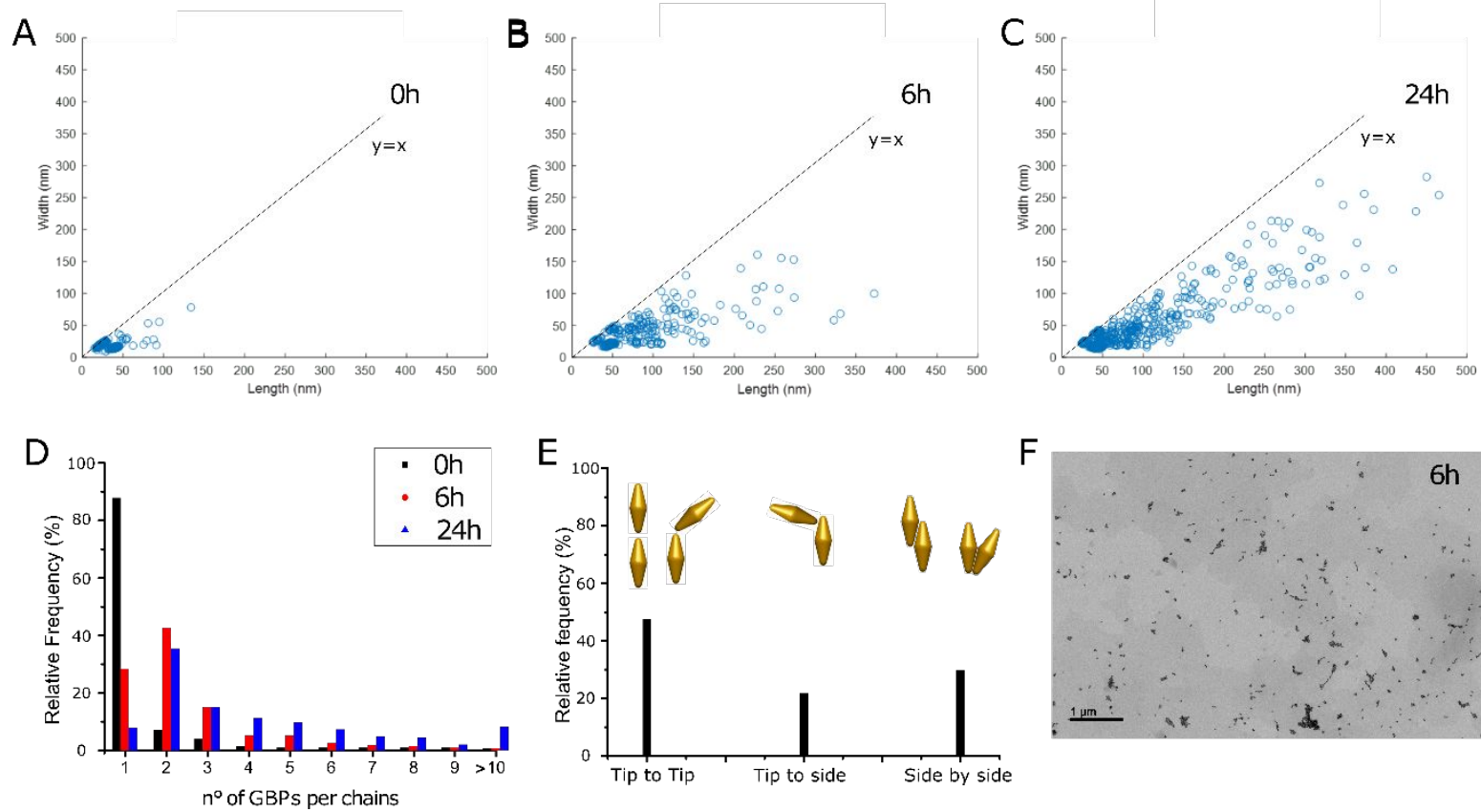

Figure S2: Statistical analysis of GBPs chains obtained at different reaction time. A-C)

Chain size distribution analyzed from TEM images at $0 \mathrm{~h}, 6 \mathrm{~h}$ and $24 \mathrm{~h}$ respectively. Each circle corresponds to a single chain. D) Statistical distribution of GBPs in assembled species after 0h (black), 6h (red) and 24h (blue). E) Relative orientation distribution in assembled species obtained after $6 \mathrm{~h}$ of reaction. F) Low magnification TEM images of GBPs chains obtained after $6 \mathrm{~h}$ of reaction. 


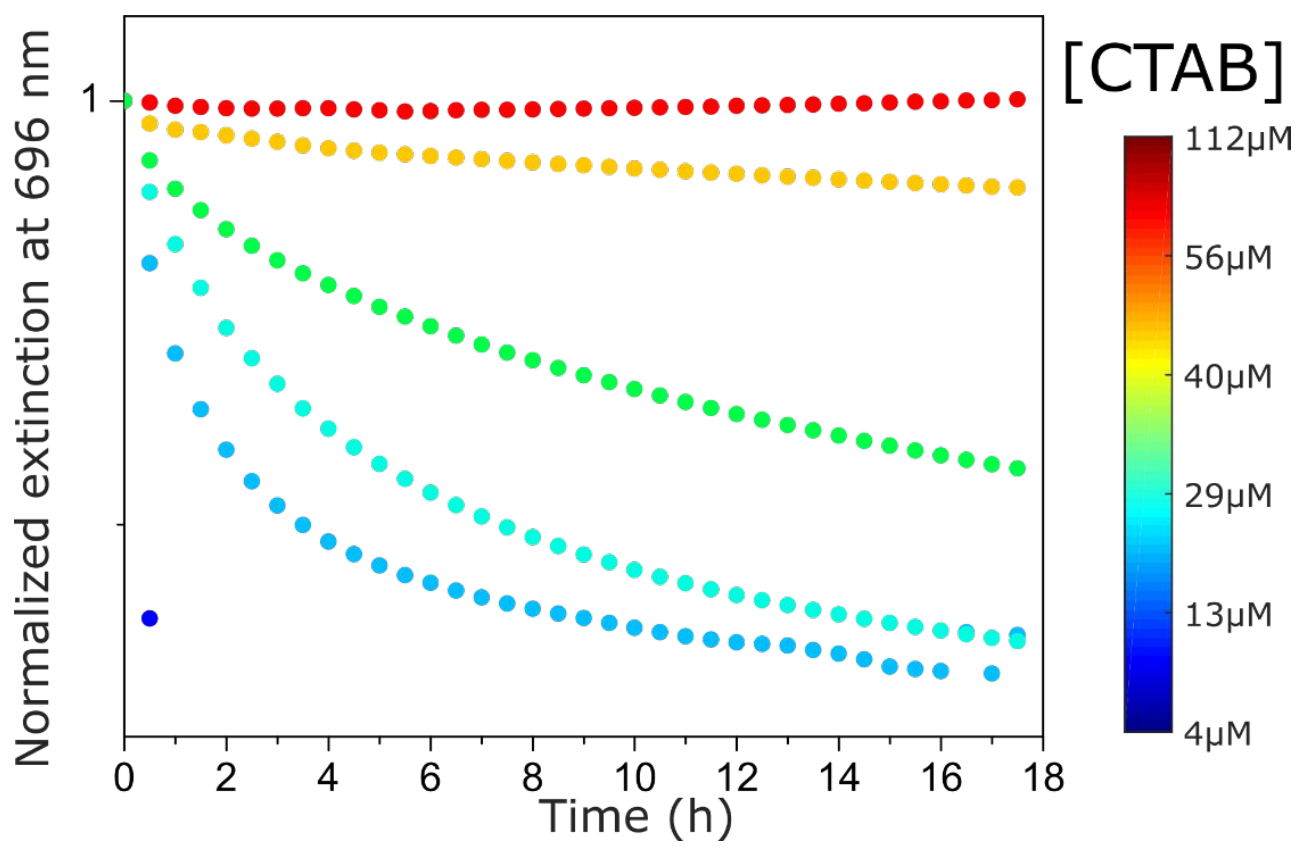

Figure S3: Tuning polymerization kinetics by adjusting CTAB concentration. Time dependence of the extinction at $696 \mathrm{~nm}$ during polymerization, for different concentrations of CTAB in the suspension.

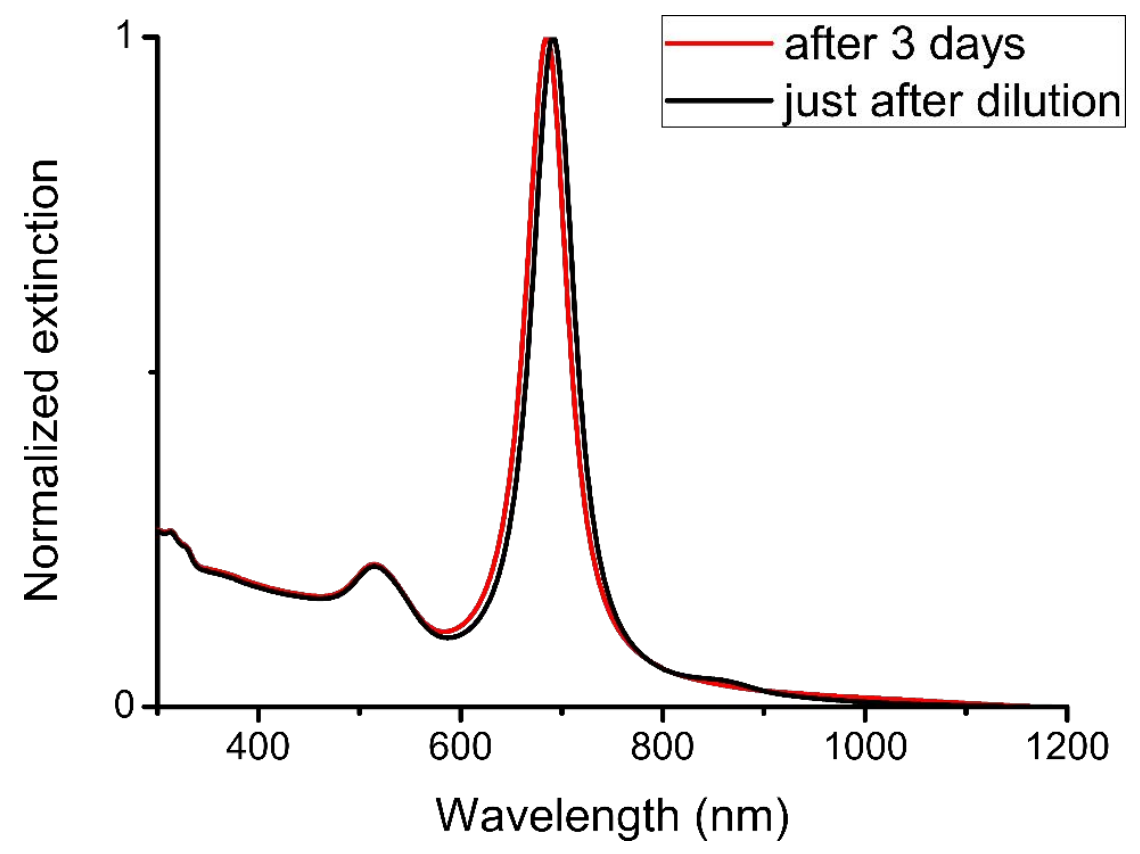

Figure S4: Stability of the GBPs diluted in water. UV/Vis spectra of the GBPs dispersion just after dilution with water (CTAB concentration of $29 \mu \mathrm{M})$ and after 3 days. 


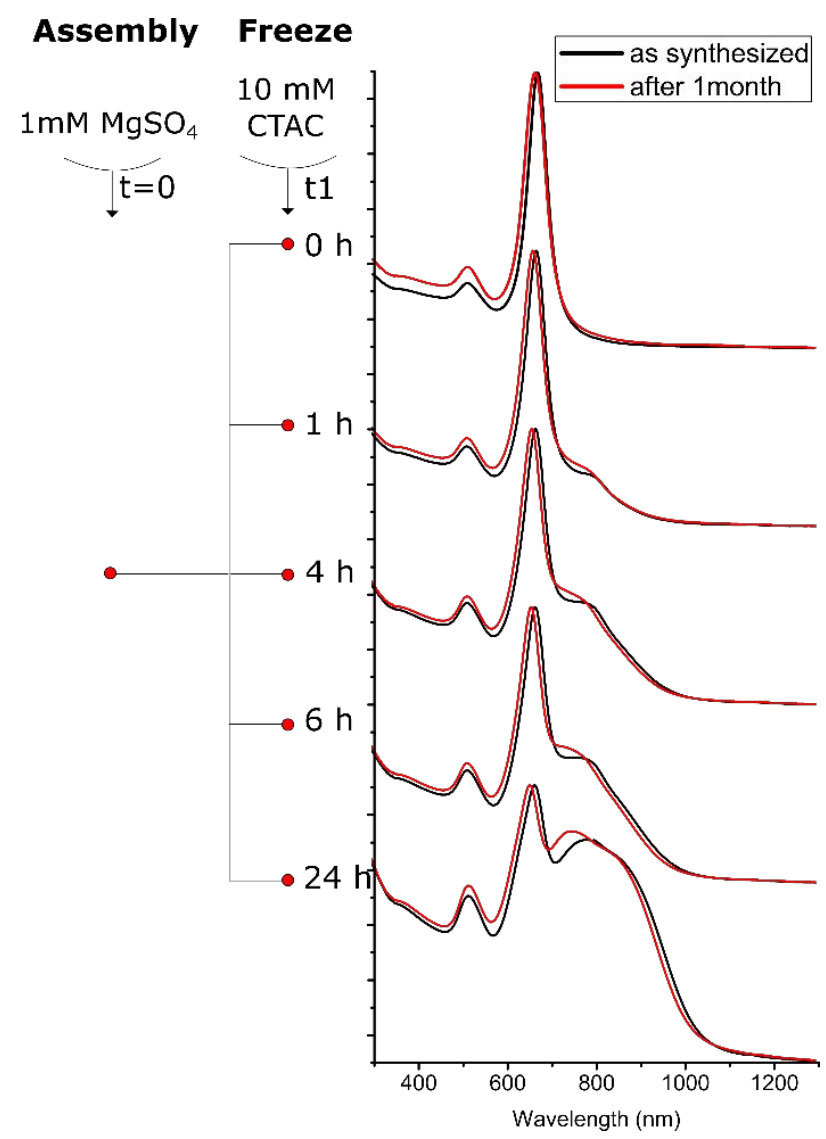

Figure S5: Controlling the degree of polymerization by freezing the self-assembly of GBPs. UV/Vis spectra of various oligomers just after freezing the self-assembly process with 10 mM CTAC surfactant mixture (black line) and the same dispersions after 1 month (red line). 

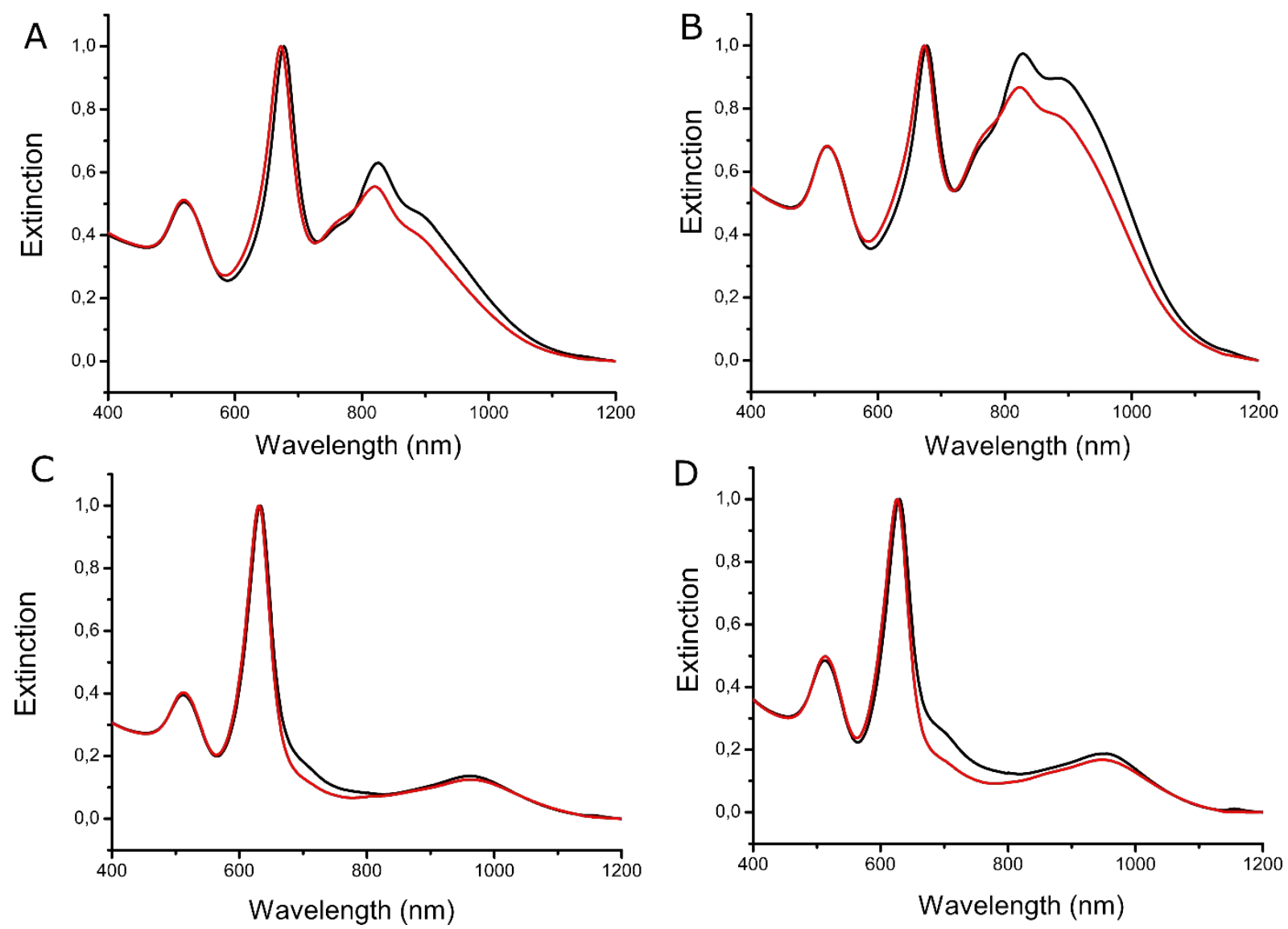

Figure S6: Stability of oligomers and connected oligomers under centrifugation. A-B)

$\mathrm{UV} / \mathrm{Vis}$ spectrum of oligomers before (black) and after (red) centrifugation and redispersion in $1 \mathrm{mM}$ CTAB. C-D) UV/Vis spectrum of connected oligomers synthesized from oligomers A) and $\mathrm{B}$ ) respectively, before (black) and after (red) centrifugation and redispersion in $1 \mathrm{mM}$ CTAB. 


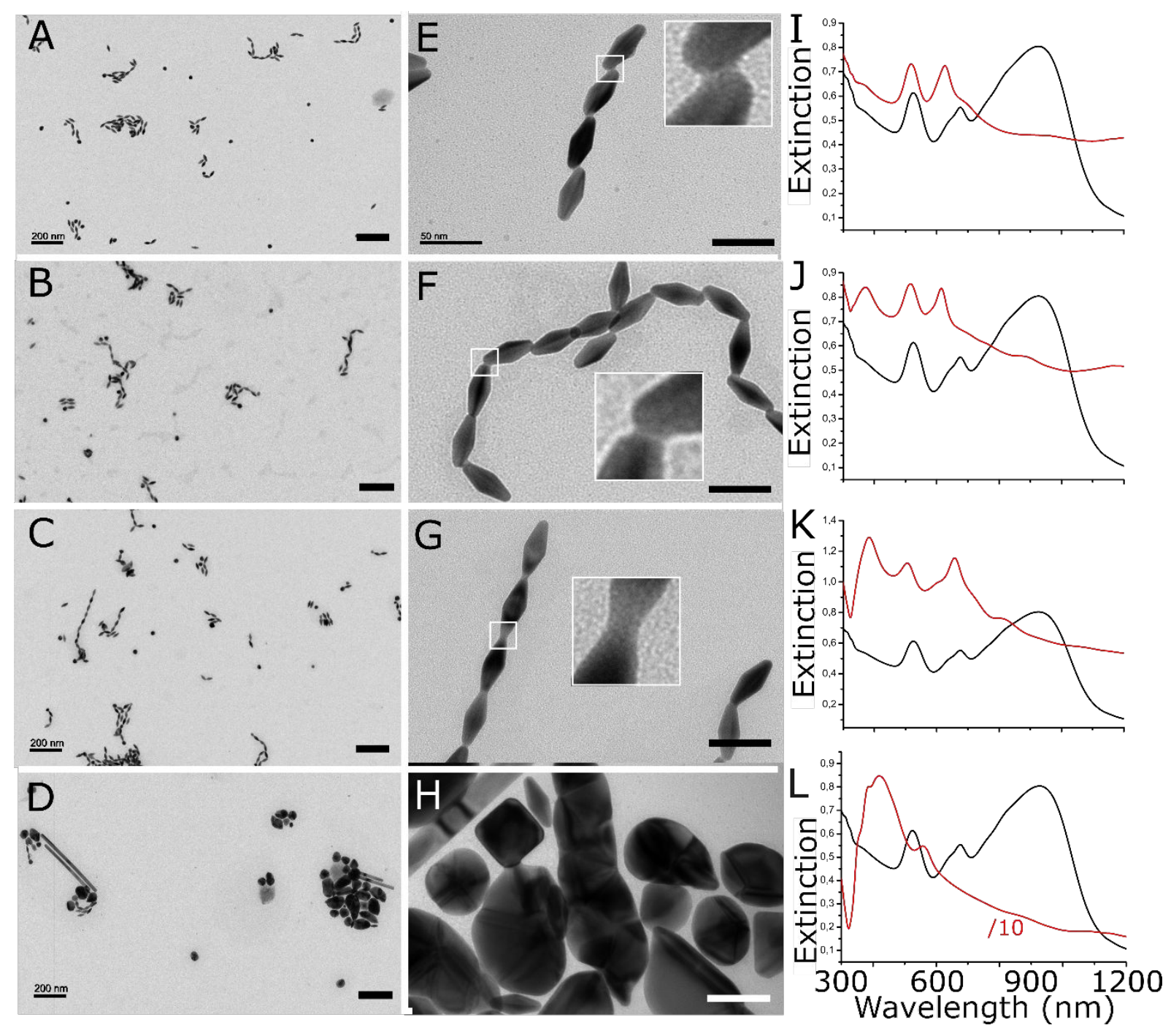

Figure S7: Effect of varying the silver quantity during synthesis of connected oligomers.

A-H) TEM images of connected oligomers prepared with various amount of Ag A, E) 0.2 eq;

B, F) 0.4 eq; C, G) 0.8 eq; D, H) 8 eq. Scale bar in A-D and E-H are $200 \mathrm{~nm}$ and $50 \mathrm{~nm}$ respectively. Insets in E-G) shows a magnified region of the conductive nanojunctions. I-L) Corresponding UV/Vis spectra of connected oligomers. Spectrum in black corresponds to the starting oligomer for comparison. Red spectrum in L) have been diluted 10 times before measurement. 


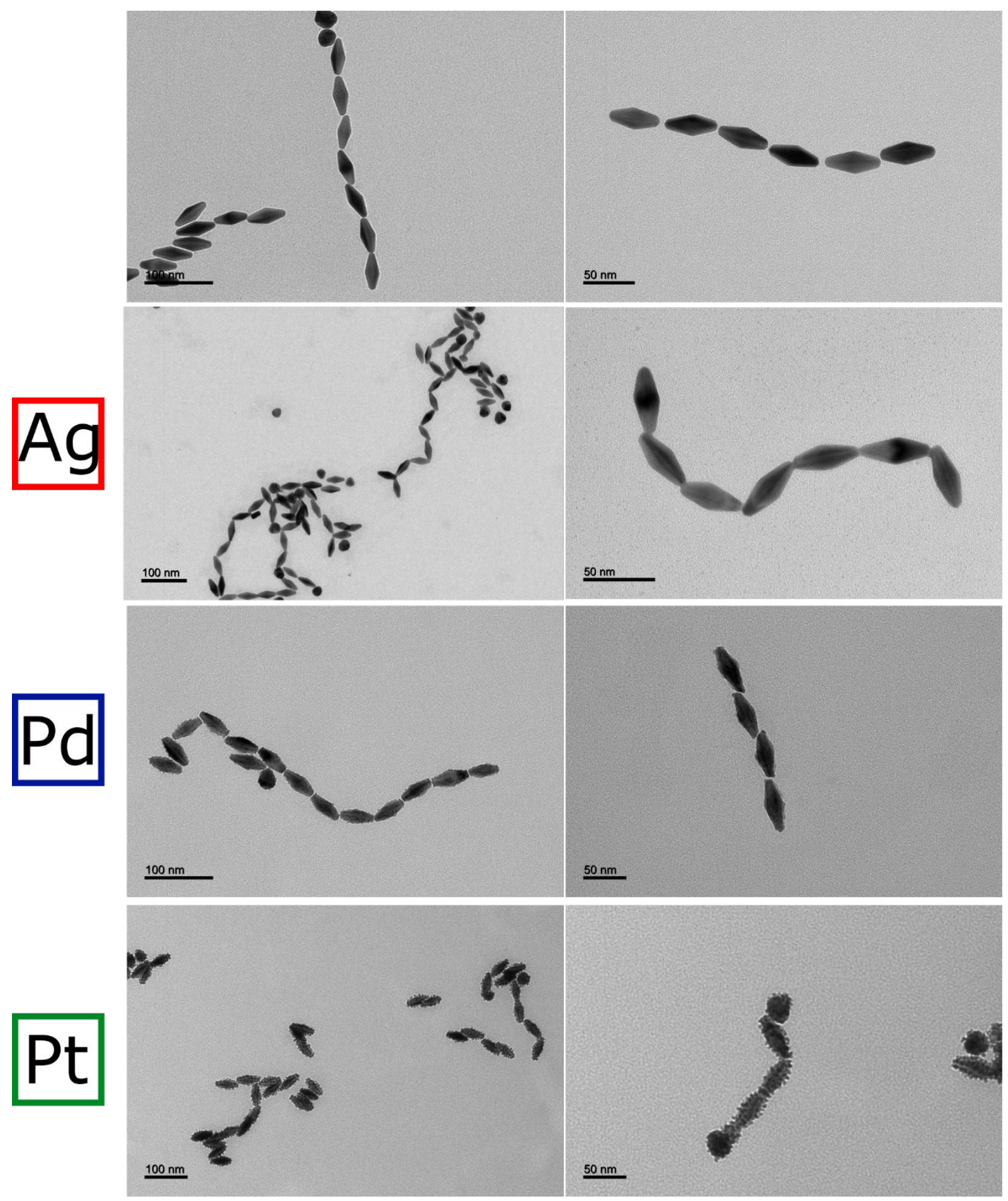

Figure S8: Additional TEM images of oligomers and connected oligomers. 

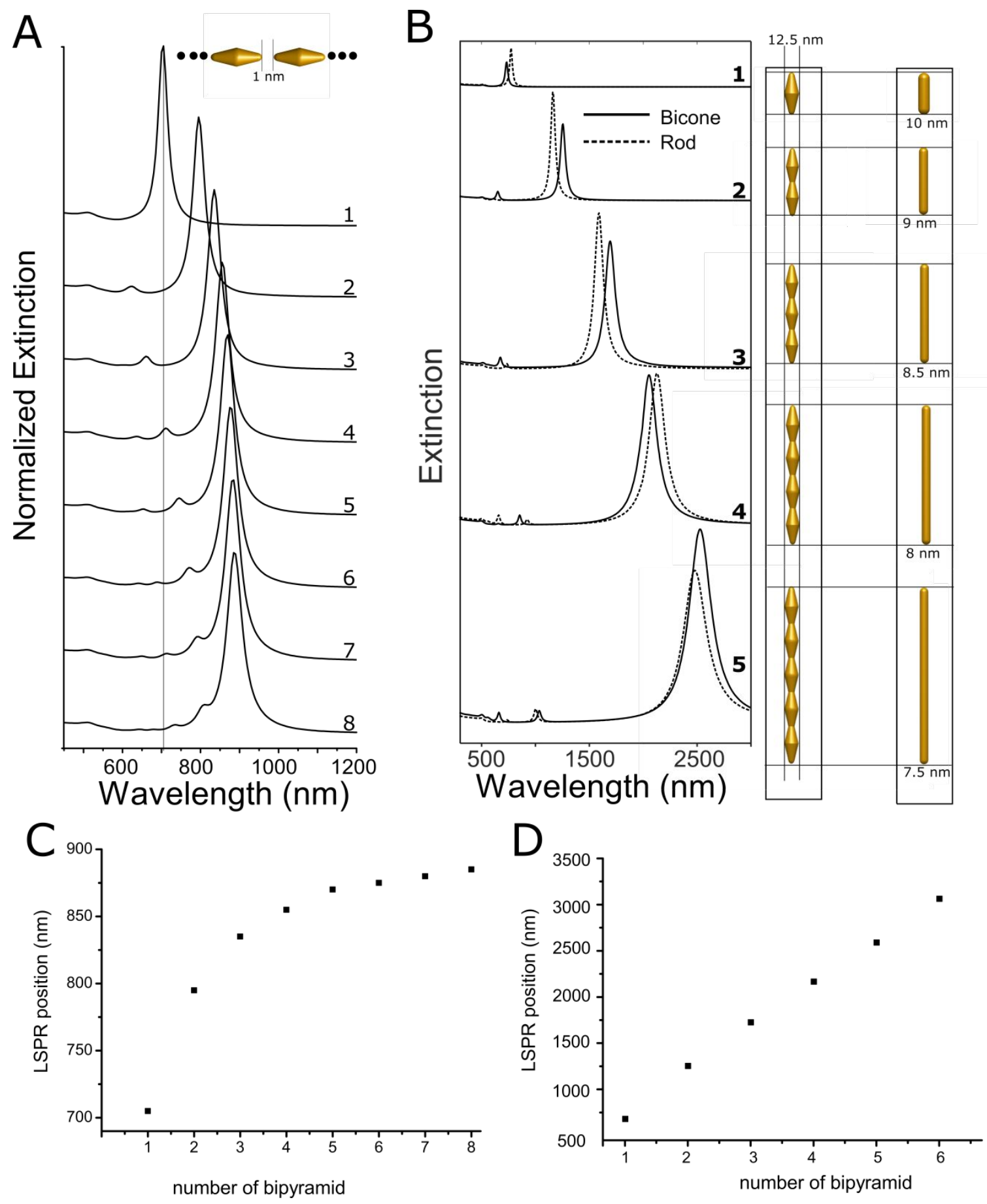

Figure S9: Calculated (BEM) extinction of oligomers and connected oligomers. A)

Oligomers, B) continuous gold nanostructure with comparison with an equivalent rod. We had to increase significantly the aspect ratio of the rods (by decreasing the diameter) to match the optical response of the corrugated particles. Indeed, the notion of aspect ratio (length/width) is no longer sufficient for describing complex particles. C-D) Graphs reporting the position of the 
dipolar L-LSPR against the number of GBPs in oligomers (C) and the continuous gold nanostructure (D).
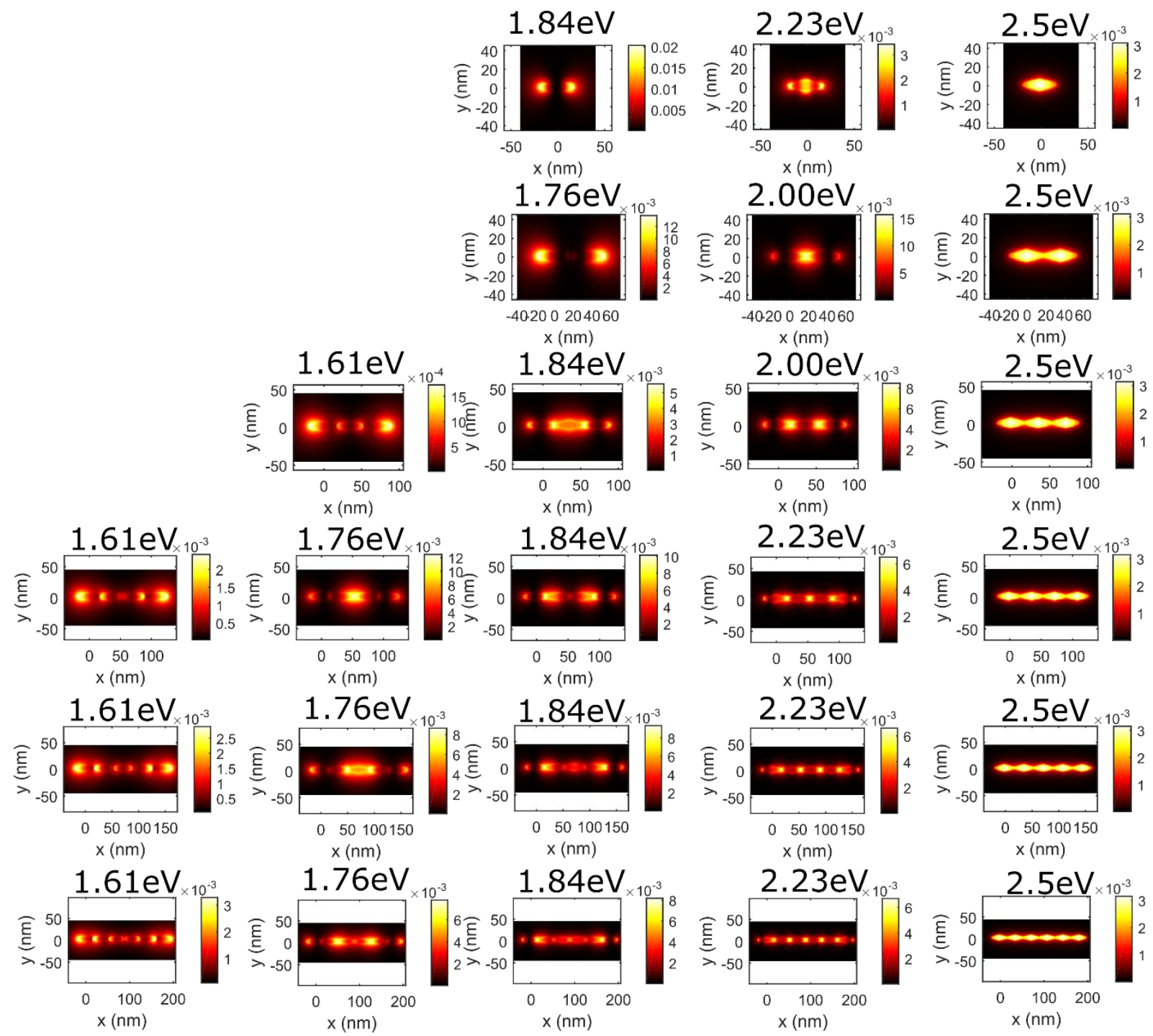

Figure S10: BEM simulations of EELS maps associated with the plasmon modes of oligomers. 

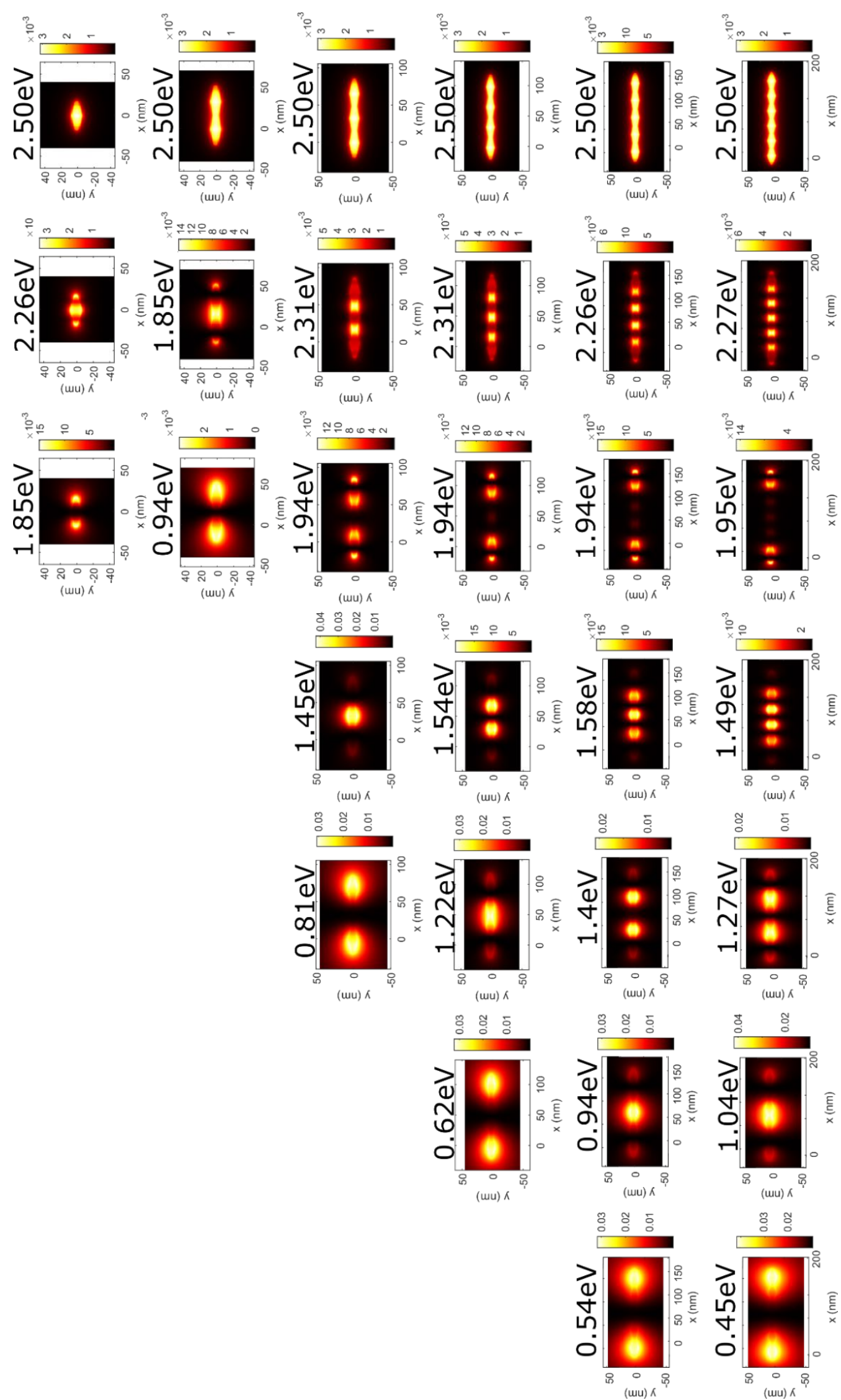

Figure S11: BEM simulations of EELS maps associated with the plasmon modes of connected oligomers. 

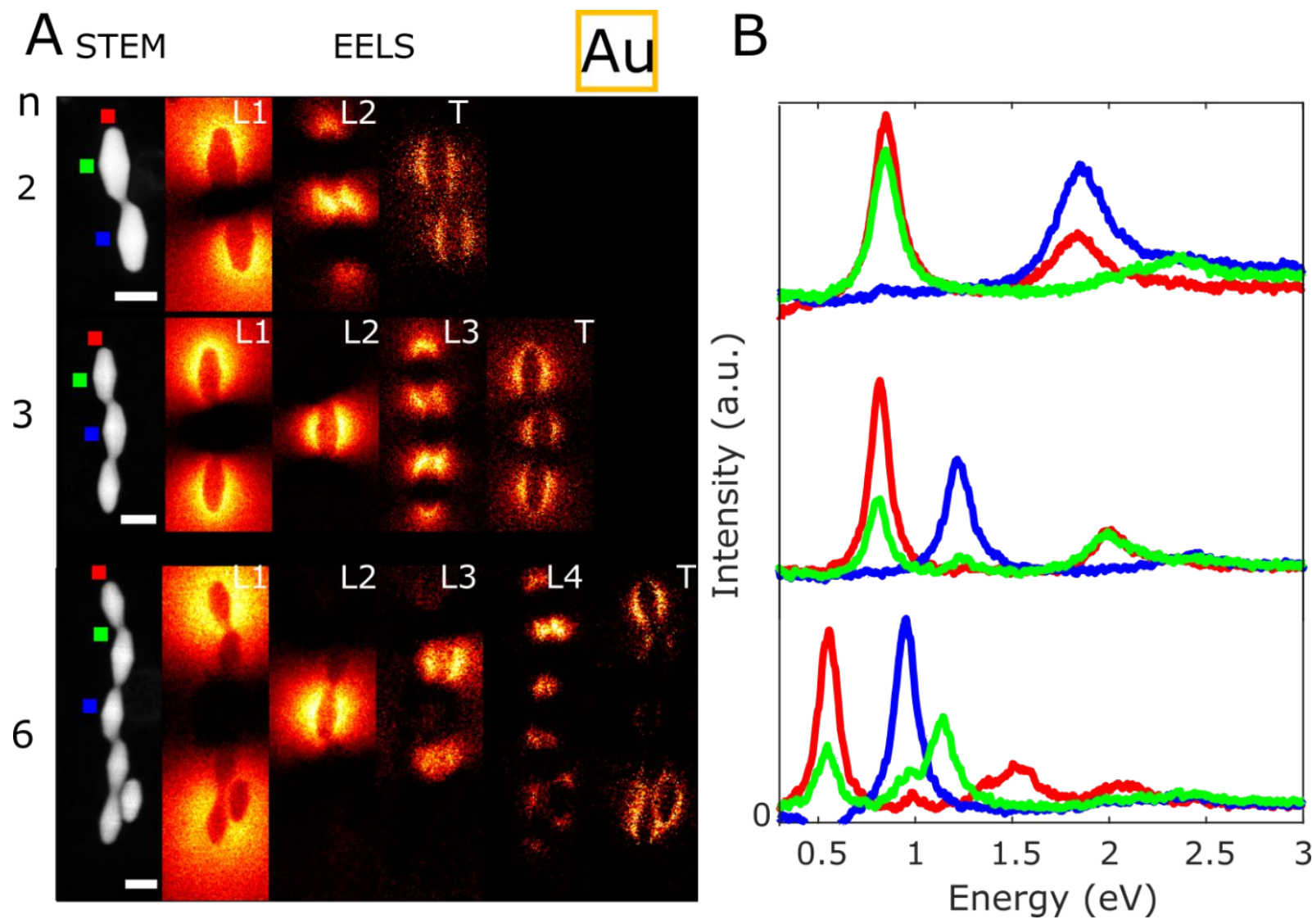

Figure S12: STEM-EELS characterization of Au connected oligomers obtained by electron beam induced welding A) Spatial distribution of plasmon modes for connected oligomers as measured by EELS; Scale bar on the STEM images is $20 \mathrm{~nm}$. EELS maps are normalized to the maximum intensity in each case. B) EELS spectra taken at the location indicated by colored squares in A). 

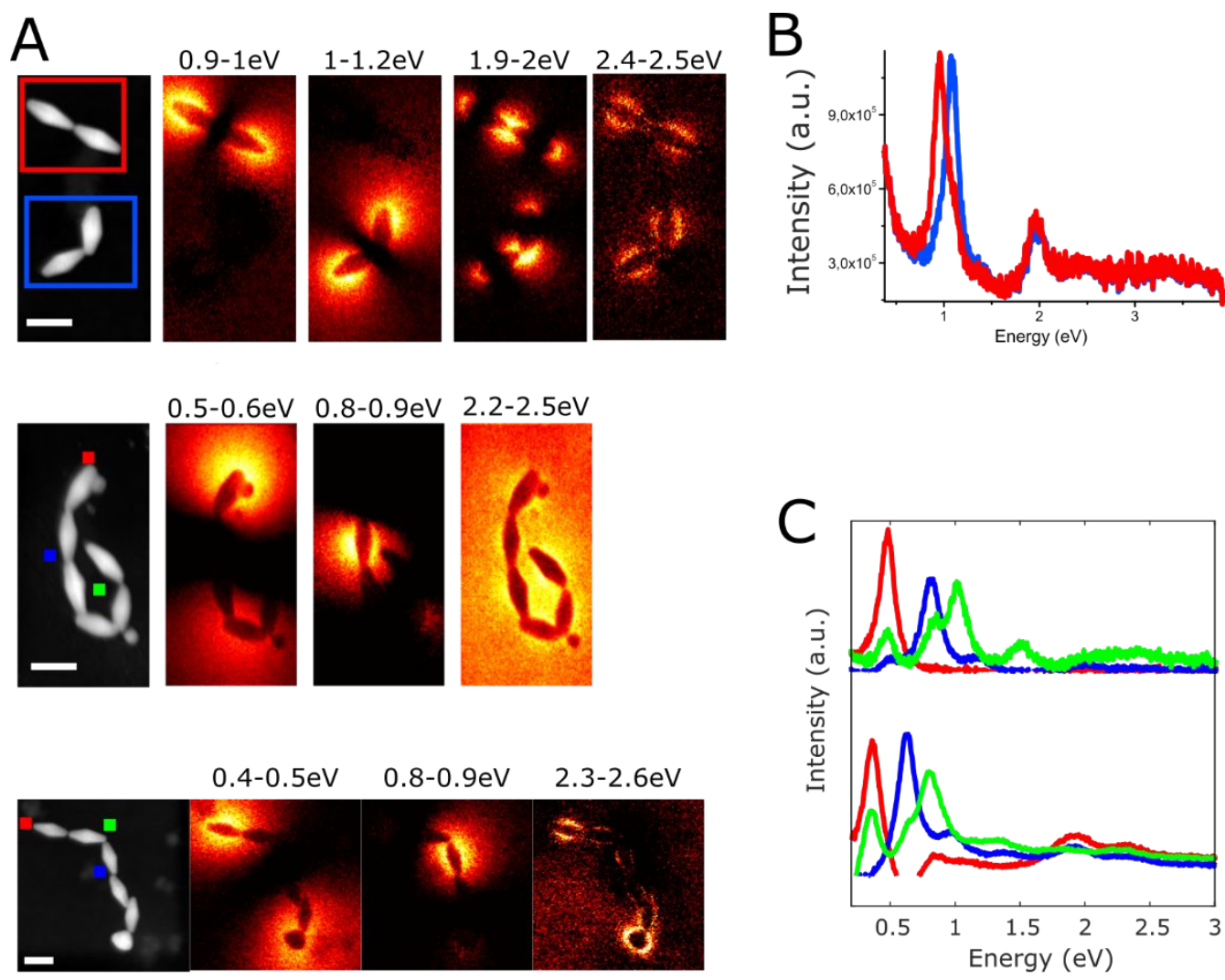

Figure S13: Effect of the oligomer symmetry on the plasmon resonance shift. A) STEM-

EELS measurements on a pair of dimer and two hexamers. Scale bar is $20 \mathrm{~nm}$. B) Corresponding EELS spectra of the dimers in the region highlighted on the STEM image. C) EELS spectra taken at the tip (red), middle (blue) and an intermediate position (green) of the connected oligomers. 


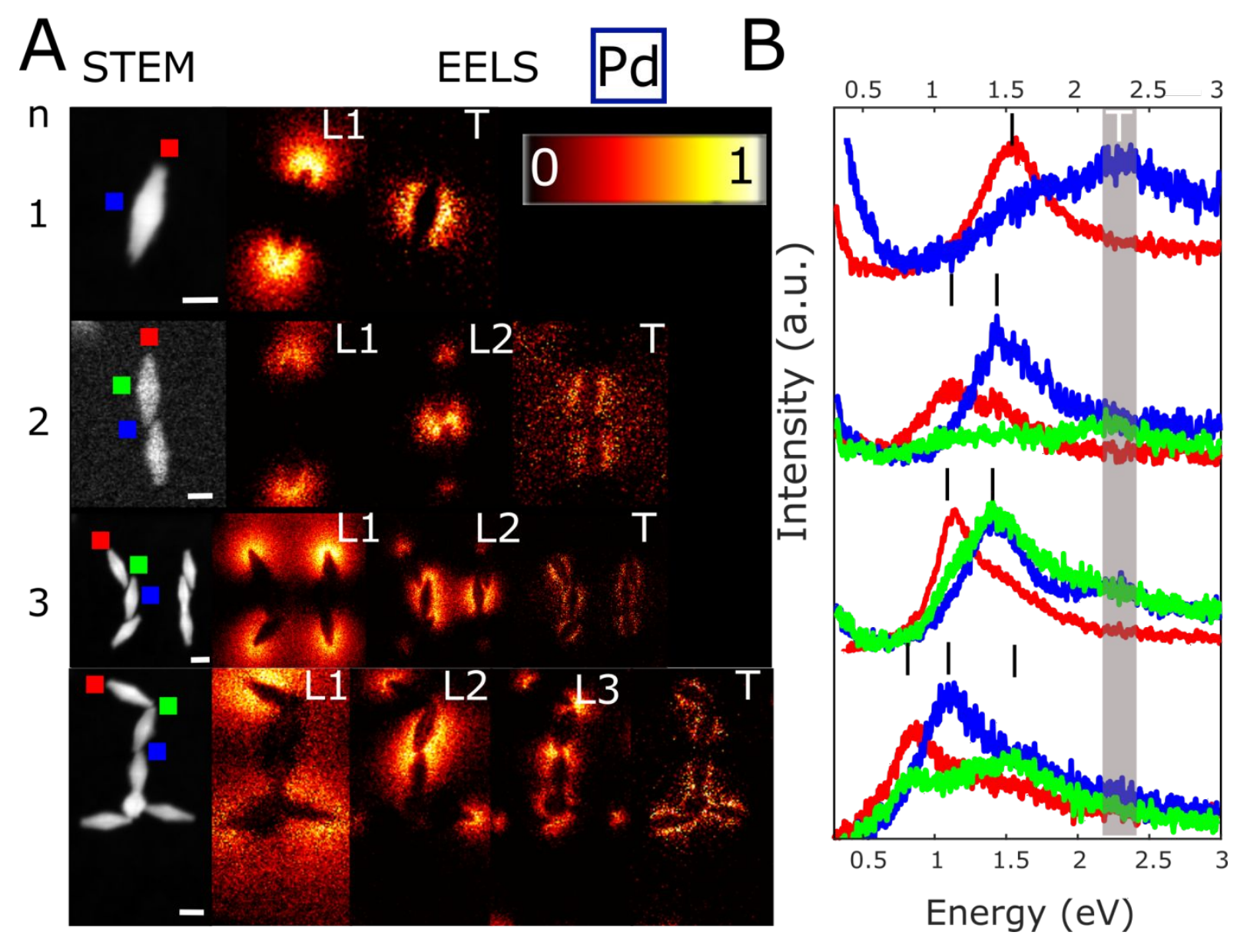

Figure S14: STEM-EELS characterization of Pd connected oligomers A) Spatial distribution of plasmon modes for connected oligomers as measured by EELS; Scale bar on the STEM images is $20 \mathrm{~nm}$. EELS maps are normalized to the maximum intensity in each case. B) EELS spectra taken at the location indicated by colored squares in A). 


\section{References}

(1) Chateau, D.; Liotta, A.; Vadcard, F.; Navarro, J. R.; Chaput, F.; Lerme, J.; Lerouge, F.; Parola, S. From gold nanobipyramids to nanojavelins for a precise tuning of the plasmon resonance to the infrared wavelengths: experimental and theoretical aspects. Nanoscale 2015, 7, 1934.

(2) Sanchez-Iglesias, A.; Winckelmans, N.; Altantzis, T.; Bals, S.; Grzelczak, M.; Liz-Marzan, L. M. High-Yield Seeded Growth of Monodisperse Pentatwinned Gold Nanoparticles through Thermally Induced Seed Twinning. J. Amer. Chem. Soc. 2017, 139, 107.

(3) Hohenester, U.; Trügler, A. MNPBEM - A Matlab toolbox for the simulation of plasmonic nanoparticles. Comp. Phys. Commun. 2012, 183, 370.

(4) Palik, E. D. In Handbook of Optical Constants of Solids; Palik, E. D., Ed.; Academic Press: Boston, 1998, p 3. 